\title{
EL ESPAÑOL EN/DESDE CHINA: A PROPÓSITO DEL EXAMEN NACIONAL EEE4
}

\author{
Anabel García \\ Antonio Becerra Bolaños
}

\section{(9) $(1) \Theta$}

Esta obra está bajo una licencia Creative Commons

Reconocimiento-No Comercial-Sin Obra Derivada 



\title{
EL ESPAÑOL EN/DESDE CHINA: A PROPÓSITO DEL EXAMEN NACIONAL EEE4
}

\author{
SPANISH IN / FROM CHINA: A PURPOSE OF NATIONAL EXAM EEE4
}

\author{
Anabel García \\ Antonio Becerra Bolaños ${ }^{1}$
}

\begin{abstract}
RESUMEN
La enseñanza del Español en China ha experimentado en estos últimos años un gran auge, tanto por el número de alumnos como por los centros de enseñanza superior que ofrecen estos estudios. El presente trabajo presenta un panorama de la enseñanza del español en los centros universitarios oficiales en China a través del análisis del Examen de Español como Especialidad EEE4, correspondiente al nivel intermedio de la lengua, y su comparación con el DELE B2 (Diploma de Español como Lengua Extranjera).

Palabras clave: Español como LE, China, examen EEE4, DELE B2, análisis de errores.
\end{abstract}

\begin{abstract}
Teaching Spanish in China has grown very rapidly in the last years both in the number of students as in the institution of higher education that offer these studies. This paper presents an overview of Spanish Teaching in official universities centres in China through an analysis of the EEE4 Test (Spanish Majors Examination level-4), corresponding to the intermediate language level, and its comparison with the DELE B2 level (Diploma of Spanish as a Foreign Language).

Key words: Spanish as a FL, China, EEE4 test, DELE B2, error analysis.
\end{abstract}

\section{Introducción}

En 1949, con el triunfo de la Revolución, el Partido Comunista Chino promovió el estudio de lenguas extranjeras por considerarlo un motor importante para su modernización. Hasta la ruptura de las relaciones sino-soviéticas, a finales de los años 50 y principios de los 60 del siglo pasado, el ruso había sido la lengua más estudiada en el país debido a la gran dependencia

\footnotetext{
M.A. Anabel García. Universidad de Jilín. Doctoranda en la Facultad de Filología China. China. Correo electrónico: anabelgarcia11@gmail.com

Dr. Antonio Becerra Bolaños. Universidad de Las Palmas de Gran Canaria. Profesor. España Correo electrónico: becerrabol@gmail.com
}

Recepción: 20- 11- 2014

Aceptación: 21- 01- 2015 
diplomática, militar, económica y técnica que existía con la URSS (Ji, 2004). A causa de la carencia de recursos y el bloqueo que sufría China por parte del mundo occidental, la antigua Unión Soviética proveía al país oriental de todos los recursos, incluidos el profesorado -entre hispanistas rusos y exiliados españoles- y los libros de texto. Yansheng (2009) subraya que, además de motivos de índole político-ideológico, como la enemistad -“China, acosada y maltratada, había de pagar con la misma moneda, rechazando todo lo procedente de los que se ensañaban en ella [sic]"-, también existía una "predisposición a confeccionar libros de texto más adecuados a la enseñanza del español en China" (p. 60).

La enseñanza del español en este país a nivel universitario apenas cuenta con 60 años de historia. No será hasta 1952 cuando, en la actual Universidad de Estudios Extranjeros de Pekín, se abra por primera vez la carrera de español. Fu Ke (1986, p. 74, citado en Ming, 2004) nos ofrece el siguiente panorama:

\begin{abstract}
En el año 1956, solo había 11 profesores de español, entre los cuales uno era profesor conferenciante y los otros, eran profesores asistentes. Desde 1953 hasta 1956 solamente se graduaron 41 alumnos de español [...] la victoria de la Revolución Cubana en el año 1959, hecho que traería un apogeo temporal de la enseñanza del español en China. Podemos verlo en la comparación de unas cifras con respecto al número de alumnos mandados a aprender idioma al exterior. Durante 1964 hasta 1966 se dispuso el gobierno central a mandar 1.547 alumnos al exterior a profundizar el estudio de idiomas, entre los cuales 414 eran para estudiar inglés, 45 para alemán, 75 para árabe mientras las personas para español eran de 240, que representaba un $16 \%$ del total personal enviado al exterior (Ming, 2004, p. 19)
\end{abstract}

La cantidad de centros universitarios oficiales en la República Popular se ha ido incrementando de manera lenta y constante hasta finales del siglo XX, si exceptuamos la época de la Revolución Cultural, entre 1966-1976 (Ji, 2004). Así, en la década de los 50 China contaba con dos centros universitarios; en los 60, con 10; en los 70, con 14; en los 80, con siete y, en 2006, con 36 (Lu, 2007). En 1986 China contaba solo con 117 alumnos de español sobre un total de 30395 estudiantes de lenguas extranjeras (Fu Ke, 1986, citado en Ming, 2004). Ya para la década de los 90, el número de alumnos por año en los centros de educación superior aumenta (Tabla 1).

Tabla 1. Alumnos matriculados en centros de educación superior china (1997-2006)

\begin{tabular}{|c|c|c|c|c|c|c|c|c|c|c|c|}
\hline Año & 1997 & 1998 & 1999 & 2000 & 2001 & 2002 & 2003 & 2004 & 2005 & 2006 & Total \\
\hline Alumnos & 135 & 192 & 269 & 305 & 350 & 360 & 455 & 510 & 696 & 1300 & 4572 \\
\hline
\end{tabular}

Fuente: Lu, 2007²/ Elaboración propia $^{3}$

Con el crecimiento de la enseñanza del español en China, se publican los primeros planes curriculares de la Licenciatura en Filología Hispánica para los cursos básicos (1998) y superiores (2000), elaborados por la Sección de Español de la Comisión Orientadora de la Enseñanza de Lenguas Extranjeras en las Universidades (Secoeleu), 《西班牙语专业教学大纲 编写组》. De acuerdo con el informe anual de la Reunión Nacional de la Enseñanza Universitaria del Español ${ }^{4}$ (全国高校西班牙语专业教学研究讨会西班牙语教学研讨年会, 2012), en 2012 el número de centros universitarios oficiales donde se enseñaba el español y que participaron en el examen nacional ascendía a 39.

En la actualidad, en las universidades oficiales chinas se ofrece la Licenciatura en Filología Hispánica, que tiene una duración de cuatro años. Desde el punto de vista de las materias que se imparten, podemos dividirla en dos ciclos de dos años cada uno: en el 
primero se le da relevancia a la adquisición de la lengua y en el segundo se profundiza más en contenidos culturales y lingüísticos. Tomamos como ejemplo el currículo de la Universidad de Jilín, si bien ciertas asignaturas pueden variar dependiendo de la institución oficial que oferte la licenciatura:

- $1^{\mathrm{o}}$ curso: Lectura intensiva de español (basado en el Español Moderno); Español básico; Conversación en español y Audición de Español.

- $2^{\circ}$ curso: Lectura intensiva de español (continuación del primer curso); Lectura extensiva de español (artículos sobre diversos temas); Conversación en español; Audición de Español; Lectura en español (incluye redacción de resúmenes y fichas de lectura); Lectura de literatura en lengua española y prácticas.

- $3^{\circ}$ curso: Lectura intensiva de español (similar a la del primer curso); Lectura extensiva de español (similar a la del segundo curso); Redacción; Lectura de periódicos españoles; Sinopsis de España (introducción general sobre geografía, historia, política, economía, etc.); Literatura de España; Sinopsis de Latinoamérica (introducción general sobre geografía, historia, política, economía, etc.); Traducción de español a chino; Interpretación de español-chino y chino-español.

- $4^{\circ}$ curso: Traducción de chino a español; Interpretación de español a chino y chino-español; Literatura de Latinoamérica; Lectura de periódicos españoles; Redacción; Conocimientos de comercio en lengua española.

Los estudiantes de la Licenciatura en Filología Hispánica ingresan sin conocimientos previos de la lengua española y, en un periodo de cuatro años, deben alcanzar un nivel equivalente al C1 y obtener un bagaje cultural acorde (Chang, 2004).

Al finalizar cada ciclo, los alumnos de segundo y cuarto cursos de los centros oficiales deben participar en un examen de carácter estatal: el examen de nivel 4 (EEE4) y el de nivel 8 (EEE8), respectivamente. La importancia de estos exámenes radica en el hecho de que los resultados que obtengan los alumnos establecen el prestigio de su facultad a escala nacional. Además, para los alumnos egresados, que obtienen una certificación expedida por la Secoeleu, serán el parámetro de referencia del nivel alcanzado para insertarse en el mercado laboral.

\section{El Examen de Español como Especialidad de nivel 4}

Después de que en 1998 la Secoeleu publicara el Programa de Enseñanza para Cursos Básicos de las Especialidades de Lengua Española en Escuelas Superiores Chinas, se convocó por primera vez (1999) el Examen de Español como Especialidad de Nivel Básico (EEE4). Este examen se realiza al término del segundo año académico. Además de establecer el nivel de conocimientos de los estudiantes, la prueba sirve a la Secoeleu para conocer la situación de cada universidad, evaluar la calidad de la educación del español y promover su desarrollo educativo.

El examen EEE4, que corresponde aproximadamente al nivel B2 del Diploma de Español como Lengua Extranjera (DELE), está dividido en dos partes. La primera está 
dedicada a la expresión escrita y la segunda, a la comprensión auditiva y expresión oral. La puntuación total del examen es de 150, de los que 100 corresponden a la parte I (Tabla 2.1), y 50, a la parte II (Tabla 2.2). La nota de corte para este examen está establecida en 90.

Tabla 2.1 Estructura del examen EEE4 (1999-2011)

\begin{tabular}{|c|c|c|c|c|}
\hline \multicolumn{5}{|c|}{ Parte 1 (100 puntos, 150 minutos) } \\
\hline \multicolumn{5}{|l|}{ Expresión Escrita } \\
\hline \multirow[b]{2}{*}{ Contenidos } & \multirow{2}{*}{$\begin{array}{l}\text { Gramática: uso } \\
\text { correcto de } \\
\text { preposiciones, } \\
\text { artículos y verbos }\end{array}$} & Gramática: & \multirow[t]{2}{*}{ Traducción } & \multirow[t]{2}{*}{ Comprensión lectora } \\
\hline & & $\begin{array}{l}\text { Corrección de } \\
\text { errores en las } \\
\text { oraciones }\end{array}$ & & \\
\hline Porcentaje/ Puntuación & \multirow{2}{*}{$38,3 \%$} & \multirow{2}{*}{$5,5 \%$} & \multirow{2}{*}{$22,8 \%$} & \multirow{2}{*}{$33,4 \%$} \\
\hline Media & & & & \\
\hline Tiempo de realización & \multicolumn{4}{|l|}{150 minutos } \\
\hline
\end{tabular}

Tabla 2.2 Estructura del examen EEE4 (1999-2011)

\begin{tabular}{|l|l|l|}
\hline \multicolumn{2}{|l|}{ Parte 2 (50 puntos, 60 minutos $)$} \\
\hline \multicolumn{2}{|l|}{ Comprensión Auditiva y Expresión Oral } \\
\hline Dic-tado & Comprensión Auditiva & Expresión Oral \\
\hline 10 ptos. & 20 ptos. & 20 ptos. \\
\hline 15 min. & 30 min. & 5 min $(+10$ min. de preparación $)$ \\
\hline
\end{tabular}

Fuente: Secoeleu (1999-2011)

En la segunda parte de la prueba, se evalúan la competencia auditiva y la expresión oral. El dictado consta de un texto que varía entre las 120 y las 150 palabras y que se repite tres veces: la primera, de manera normal y las siguientes, a menor velocidad. Para la comprensión auditiva, se establecen las siguientes pautas:

a. Compresión del material auditivo de nivel intermedio cuyas palabras nuevas no superen el 3\% de las palabras en total y una velocidad del habla entre 100 y 120 palabras por minuto.

b. Compresión del material auditivo sobre temas de vida diaria y social o vida de los países hispanohablantes.

Para ello, se ha de escuchar un texto de 500 palabras aproximadamente. La grabación se repite tres veces y luego se plantean 10 preguntas, que se repiten una vez, formuladas en la misma grabación sobre el texto. Tras el dictado y la comprensión auditiva, el alumno deberá grabar un monólogo de cinco minutos sobre el tema sugerido por la mesa examinadora (Tabla 2). Los temas propuestos para este apartado del EEE4 han ido variando de acuerdo con la realidad nacional y merecerían un análisis más profundo (Tabla 3). 
Tabla 3. Temas del apartado de expresión oral del EEE4 (1999-2013)

\begin{tabular}{|c|c|}
\hline Año & Tema \\
\hline 1999 & Sobre tu tierra natal. \\
\hline 2000 & Sobre la maestra o el maestro que te enseñó las primeras letras. \\
\hline 2001 & ¿Qué opinas acerca de la publicidad? ¿Es beneficioso o perjudicial? \\
\hline 2002 & ¿Qué quieres ser cuando termines la carrera de español? \\
\hline 2003 & Habla de un amigo tuyo o de una amiga tuya. \\
\hline 2004 & ¿Cuál es tu profesión favorita? ¿Por qué? \\
\hline 2005 & ¿Por qué has elegido el español como tu carrera? ¿Qué piensas ahora sobre tu elección? \\
\hline 2006 & ¿Cuál de las cuatro estaciones del año es la que más te gusta? ¿Por qué? \\
\hline 2007 & Un viaje o una excursión inolvidable. \\
\hline 2008 & ¿Qué haces en tu tiempo de ocio? \\
\hline 2009 & Cuenta la experiencia de una fiesta organizada entre amigos. \\
\hline 2010 & ¿Qué opinas sobre Internet? \\
\hline 2011 & ¿Ayudar a otros te hace sentir alegre? ¿Estás de acuerdo con esta opinión? \\
\hline 2012 & ¿Por qué cada vez se aprende más el chino? \\
\hline 2013 & Las bicicletas en nuestro país. \\
\hline
\end{tabular}

Fuente: Secoeleu

El vocabulario y las estructuras gramaticales del EEE4 son tomados del libro de estudio común de la mayoría de las universidades chinas; nos referimos al Español Moderno, de Dong Yansheng, cuyos seis tomos se publicaron entre 1999 y 2007. El objetivo de Yansheng era paliar los problemas lingüísticos que los sinohablantes tenían en el proceso de adquisición de la lengua y que los manuales españoles o hispanoamericanos no tomaban en cuenta. Ello se debía, explica el propio Yansheng (2009), a que contemplaban a hablantes de lenguas indoeuropeas, cuyas diferencias con el chino son "casi abismales" (p. 61).

No hay que olvidar que Sánchez-Griñán (2008) había señalado la ausencia de consideraciones pedagógicas en la enseñanza de lenguas extranjeras en China como uno de sus principales problemas:

En las universidades, los estudios de lengua española están programados según un plan rígido, ajeno a la reflexión didáctica. El grado de simplicidad y complejidad de las nociones gramaticales es suficiente criterio para ordenar un corpus, materializado en libros de texto como Español Moderno [...]. Las funciones comunicativas se introducen ocasionalmente, según su gramática se haya dado o no; se encuentran, pues, al servicio de la secuenciación gramatical. No hay ninguna indicación acerca de la inclusión gradual de las mismas, pues se asume que su aprendizaje resultaría como consecuencia natural del estudio e interiorización de las reglas gramaticales, que a su vez solo es posible como resultado de la formación de hábitos lingüísticos (p. 97) 
Esta idea es la que defiende, precisamente, Yansheng (2009), que afirma: "Las preguntas que hago cumplen un doble cometido: comprobar si se ha entendido el contenido y ejercitar al alumnado en los ítems gramaticales y léxicos que corresponden a la lección” (p. 64).

Para entender el estadio en que se encuentra el español en China, debemos señalar que históricamente su estudio es muy reciente y la demanda de profesores se ha incrementado exponencialmente, un gran número de ellos no cuenta con la preparación pedagógica, metodológica y lingüística requerida. El Español moderno, al ser "el centro del examen", asegura a los docentes, por un lado, una herramienta sólida para no "cometer errores" durante la enseñanza y, por otro, la preparación certera para el EEE4.

Como ya advirtiera Sánchez-Griñán (2008, 2009), nos encontramos ante un modelo pedagógico basado en la figura del profesor y en una enseñanza que tiende a la repetición de las estructuras gramaticales. Los alumnos, basándose en la información teórica del libro, pueden prepararse para el examen y superarlo. Sánchez-Griñán (2008) había advertido cómo este sistema de enseñanza de lenguas extranjeras "está marcado por la continuidad de un esquema cultural donde convergen elementos del confucionismo y elementos de una tradición pragmatista que da especial importancia al sistema de exámenes y a la traducción" (p. 92). No obstante, ello no debe de ser tomado como un defecto por cuanto, como tendremos la ocasión de comprobar, ha cumplido con los fines de la Secoeleu. En este sentido, Arriaga, Blanco y Lamarti (2012) critican precisamente esa tendencia a juzgar el libro de Yansheng sin comprender la función que tiene en el contexto chino.

\subsection{Evaluación, evolución y resultados del EEE4}

La Secoeleu es la institución responsable del examen y las universidades de Pekín, Nankín y de Estudios Internacionales de Shanghai participan en la elaboración de los contenidos y su evaluación. Las universidades, por su parte, se encargan del registro de sus estudiantes y de la ejecución del examen anual. Los resultados obtenidos por los centros universitarios se analizan en la Reunión Nacional de la Enseñanza Universitaria del Español, que se efectúa cada año con el propósito de promover la ejecución del plan marcado por la Secoeleu.

Sabemos que, en 1999, China contaba con 11 universidades con facultad de Español. Eran las universidades más prestigiosas como la Universidad de Pekín, la Universidad de Estudios Extranjeros de Pekín, la Universidad de Estudios Internacionales de Shanghai, entre otras, consideradas de primera clase y que contaban con los alumnos de notas más altas en la selectividad. En 2006, el número de centros oficiales se situaba en 36, con unos 4000 estudiantes matriculados (Lu, 2007) y, en 2010, había 6000 entre matriculados en Filología Hispánica y estudiantes de Español como segunda lengua (Marco-Martínez y Lee-Marco, 2010).

El ascenso en el número de alumnos que se presentaban al EEE4 se puede apreciar en la evolución de los resultados. Así, durante el periodo comprendido entre 1999 y 2011 (Tabla 4), se puede observar que la variación de la calificación más alta se ha mantenido constante, lo que no sucede con el promedio y la nota más baja, que han experimentado un descenso del 15,6\% y 43,6\%, respectivamente. Carecemos de datos sobre el número de alumnos que participaron en estos años de forma total, aunque, según Lu (2007), en 1999 se habían presentado 125 alumnos (redELE, 2012) por los 2647 que se presentaron en 2012 (Tabla 5). El descenso en las notas, por tanto, está relacionado con el aumento exponencial del número de alumnos y de centros en los que se imparte el español, tanto como especialidad como L2, en estos últimos años. 
Tabla 4. Notas obtenidas en el EEE4 entre 1999 y 2011

\begin{tabular}{|l|l|l|l|}
\hline Año/Nota & Más baja & Promedio & Más alta \\
\hline $\mathbf{1 9 9 9}$ & 66,35 & 108,33 & 136,35 \\
\hline $\mathbf{2 0 0 0}$ & 53,10 & 105,09 & 139,15 \\
\hline $\mathbf{2 0 0 1}$ & 58,60 & 103,56 & 136,20 \\
\hline $\mathbf{2 0 0 2}$ & 16,75 & 96,48 & 133,75 \\
\hline $\mathbf{2 0 0 3}$ & 25,15 & 91,09 & 138,43 \\
\hline $\mathbf{2 0 0 4}$ & 29,55 & 110,04 & 143,35 \\
\hline $\mathbf{2 0 0 5}$ & 14,80 & 97,25 & 137,20 \\
\hline $\mathbf{2 0 0 6}$ & 29,40 & 104,35 & 136,41 \\
\hline $\mathbf{2 0 0 7}$ & 7,05 & 97,06 & 132,10 \\
\hline $\mathbf{2 0 0 8}$ & 8,35 & 96,29 & 141,20 \\
\hline $\mathbf{2 0 0 9}$ & 5,55 & 83,82 & 132,55 \\
\hline $\mathbf{2 0 1 0}$ & 8,25 & 90,33 & 137,95 \\
\hline $\mathbf{2 0 1 1}$ & 1 & 85,03 & 136,55 \\
\hline
\end{tabular}

Fuente: Secoeleu (2011)

En 2012, 39 universidades oficiales participaron en el EEE4, con un número de alumnos de 2647, de los cuales 1917 lo hacían por primera vez. De estos 1917, 820 aprobaron $(42,7 \%)$, mientras que se desconoce el número de aprobados sobre los restantes presentados (Tabla 5).

Tabla 5. Número de alumnos presentados y aprobados

\begin{tabular}{|l|l|l|l|}
\hline Año & Participantes EEE4 & Aprobados & Total universidades \\
\hline 2007 & 646 & 462 & \\
\hline 2008 & & & \\
\hline 2009 & 1000 & 418 & $23^{4}$ \\
\hline 2010 & 1124 & 634 & \\
\hline 2011 & 1543 & 715 & \\
\hline 2012 & $2647(1917)$ & 820 & 39 \\
\hline
\end{tabular}

Fuente: Socoeleu $(2007,2009,2010,2011,2012)$

\subsection{El EEE4 y el DELE B2}

La estructura del EEE4 tiene como objetivo evaluar el conocimiento de los estudiantes que han concluido el segundo curso en instituciones universitarias. Un análisis de su contenido nos permite definir las destrezas que se consideran importantes como resultado de la enseñanza en el sistema chino. Para visualizarlos mejor, hemos establecido una comparación entre el EEE4 y el nivel B2 del DELE, en tanto que ambos corresponden al nivel intermedio de conocimiento del idioma. El DELE, creado en el contexto del Marco común europeo de referencia para las lenguas (Consejo de Europa, 2002), es un referente sólido ya que intervienen en su elaboración expertos en pedagogía y lingüística aplicada, y por la tradición de enseñanza y adquisición de lenguas debido a la diversidad lingüística de la región. 
Tabla 7. Comparación EEE4 y DELE B2

\begin{tabular}{|c|c|}
\hline EEE4 & DELE B2 \\
\hline \multirow[t]{2}{*}{$\begin{array}{l}\text { Parte I ( } 150 \mathrm{~min} .) \\
\text { 1. Uso correcto de preposiciones, artículos y verbos } \\
\text { 2. Corrección de errores en las oraciones. } \\
\text { 3. Traducción ( } 10 \text { oraciones) } \\
\text { 4. Comprensión de lectura }\end{array}$} & $\begin{array}{l}\text { Parte I }(180 \text { min.) } \\
\text { 1. Comprensión de lectura }(60 \mathrm{~m}) \text {. Elección } \\
\text { múltiple }(\mathrm{EM}) \\
\text { 2. Expresión escrita }(60 \mathrm{~m}) \text {. Redacción de un texto } \\
\text { entre } 150 \text { y } 200 \text { palabras. }\end{array}$ \\
\hline & $\begin{array}{l}\text { Parte II ( } 90 \text { min.) } \\
\text { 3. Comprensión Auditiva }(30 \mathrm{~min} .)(\mathrm{EM} / 2 \text { veces }) \\
\text { 4. Gramática y vocabulario }(60 \mathrm{~m})(\mathrm{EM})\end{array}$ \\
\hline $\begin{array}{l}\text { Parte II }(60 \mathrm{~min} .) \\
\text { 1. Dictado }(15 \mathrm{~min} .) \\
\text { 2. Comprensión auditiva }(30 \mathrm{~min} .) \\
\text { 3. Expresión oral }(5 \mathrm{~min}+10 \mathrm{~min} \text { preparación }) \\
\text { (grabación monólogo) }\end{array}$ & $\begin{array}{l}\text { Parte III } \\
\text { 5. Expresión Oral (10/15m + Preparación } 10 \text { min.) } \\
\text { (Interacción con examinador) }\end{array}$ \\
\hline
\end{tabular}

Fuente: Secoeleu / Instituto Cervantes (2007)

Tal como muestra la tabla 6, estructuralmente el EEE4 consta de dos partes, mientras que el DELE B2 contempla tres, si consideramos que la comprensión de lectura y la expresión escrita, por un lado, y la comprensión auditiva y gramática y vocabulario, por otro, forman dos bloques de pruebas por separado. El EEE4 tiene una duración total de 210 minutos, mientras que el DELE B2, de 250 minutos aproximadamente, dependiendo del tiempo que dure la interacción entre el alumno y el examinador. Otra diferencia radica en el hecho de la evaluación: en el EEE4 no se ha de obtener una mínima en cada uno de los apartados, sino que basta con obtener un total de 90 puntos para superar la prueba.

Además, el tratamiento que se da a las cuatro competencias presenta algunas diferencias. En la expresión escrita, el EEE4 no contempla la redacción, algo que se suple al proponer al alumno ejercicios de respuesta abierta en las dos partes de la prueba. Por su parte, el DELE B2 incluye un apartado de redacción, pero el resto de los ejercicios es de elección múltiple.

En la comprensión auditiva, mientras en el EEE4 se realiza la audición de un solo texto, que es repetido tres veces y sobre el cual se hacen 10 preguntas orales abiertas que se repiten una sola vez, en el DELE B2 se ofrecen cuatro textos orales, repetidos dos veces, sobre los cuales se plantean tres preguntas de elección múltiple. Por último, en la expresión oral, en el EEE4 no existe interacción alguna con los examinadores. Se le propone un tema al alumno, el cual tiene 10 minutos para prepararse y cinco para grabar su exposición. Esta se realiza a través de una grabación en una cinta que será evaluada por la comisión oficial.

\subsection{Análisis de los errores de los estudiantes chinos en el EEE4}

Si observamos las calificaciones de los estudiantes chinos en el examen de 2011 (Tabla 7), la nota promedio de la interpretación, el dictado y la compresión auditiva no llega a la mitad de la nota total, por lo que son las partes en que suelen errar. Hay que considerar que los tres primeros tomos del Español Moderno sirven "para enseñar a hablar, leer y escribir en español" a través de un "entrenamiento intensivo en el primer año y medio para conseguir recursos expresivos elementales" (Yansheng, 2009, p. 63). 
Tabla 8. Examen de EEE4 del año 2011

\begin{tabular}{|l|l|l|}
\hline & Nota total & Nota promedio \\
\hline Examen Escrito & $\mathbf{1 0 0}$ & $\mathbf{6 2 , 1 5}$ \\
\hline 1 Preposiciones y artículos & 10 & 6,73 \\
\hline 2 Verbos & 20 & 12,12 \\
\hline 3 Selección de palabras correctas & 6,00 & 4,06 \\
\hline 4 Corrección de errores en oraciones & 9,00 & 5,36 \\
\hline 5 Traducción del chino al español & 22,00 & 15,18 \\
\hline 6 Comprensión lectora & 33 & 18,71 \\
\hline $\begin{array}{l}\text { 6.1 Indicar a qué se refieren las palabras subrayadas y el sujeto de } \\
\text { los verbos }\end{array}$ & 10 & 6,41 \\
\hline 6.2 Seleccionar la opción más adecuada según el texto & 5 & 3,62 \\
\hline 6.3 Interpretación & 10 & 2,95 \\
\hline 6.4 Traducción del español al chino & 8 & 5,73 \\
\hline Examen Oral & $\mathbf{5 0}$ & $\mathbf{2 2 , 8 6}$ \\
\hline 1 Dictado & 10 & 4,68 \\
\hline 2 Compresión auditiva & 20 & 6,11 \\
\hline 3 Composición oral & 20 & 12,11 \\
\hline
\end{tabular}

Fuente: Secoeleu/Elaboración propia

En el caso del texto de Interpretación que fue objeto de esta prueba en el EEE4 de 2011, se propuso el tema del paso del tiempo. Los estudiantes tuvieron que interpretar en español las siguientes oraciones o las partes subrayadas:

1. [...] esa locura de restauración de edificios de importancia, que se ha apoderado de muchos Ayuntamientos y que financian los ciudadanos.

2. No llego a entender a qué se debe esta obstinación por detener el deterioro de unas figuras que fueron diseñadas para vivir al aire libre, [...]

3. [...], porque es demasiado lo que se expone en los museos como para poder ser contemplado con calma y detenimiento.

4. Todo esto se hace con nuestro dinero y no estamos precisamente fuera del peligro de carencias y necesidades.

5. [...] es inaceptable el paso del tiempo, neguémoslo con convicción, cueste lo que cueste.

Tabla 9. Porcentaje de notas de los estudiantes

\begin{tabular}{|l|l|l|l|l|}
\hline Notas ( total 10 puntos) & $0-2,5$ & $2,75-5$ & $5,25-8$ & $8,25-10$ \\
\hline Número de estudiantes (total 2027) & 1104 & 501 & 360 & 62 \\
\hline Porcentaje & $54,44 \%$ & $24,78 \%$ & $17,72 \%$ & $3,06 \%$ \\
\hline
\end{tabular}

Fuente: Secoeleu/Elaboración propia

Entre los 2027 estudiantes que participaron en el EEE4 de 2011, más de la mitad $(54,44 \%)$ obtuvo una nota inferior a 2,5 puntos. Los errores en la interpretación que cometieron se debían a problemas comunes, en el ámbito de la cohesión (Zhi, 2009): 
- Problemas gramaticales y ortográficos como conjugación de verbos, uso del subjuntivo, confusión de género y número, etc.

- Confusión del sujeto de las oraciones compuestas, lo cual contribuye a una mala comprensión.

- Vocabulario limitado, por lo que no conocen las palabras o no pueden expresar lo que piensan de una manera apropiada.

- En los textos suelen aparecer frases hechas y expresiones fijas que son difíciles de entender.

El dictado de la misma prueba estaba compuesto por un texto de 127 palabras con 15 signos de puntuación. En las dos primeras oraciones del texto (1 y 2), se produjeron errores significativos (Secoeleu, 2011).

1. "La vida moderna (1) no (2) exige (3) actividad (4) física (5). (6)"

(1) modena, mordena

(2) nos

(3) exije, exgijimos, ex, exijer, existe

(4) activadad, activida, tipid, tividad, tividades

(5) físico

(6) ausencia del punto final

2. "Cogemos (1) el (2) coche para ir a trabajar, (3)"

(1) gogemos, cojemos, cojimos

(2) en el

(3) falta de coma

Tabla 10. Porcentaje de cada tipo de error en el dictado

\begin{tabular}{|l|l|l|}
\hline Problemas fonéticos & Problemas de gramática & Problemas de ortografía \\
\hline $82,52 \%$ & $13,50 \%$ & $3,98 \%$ \\
\hline
\end{tabular}

Fuente: Secoeleu (2011) / Elaboración propia

Los problemas fonéticos que tienen los estudiantes chinos $(85,52 \%)$ ya habían sido señalados como comunes por Yansheng (2009) y consisten en la confusión entre los fonemas y las grafías: /p/, /t/, /k/, /b/, /d/,/g/, /l/, /r/, / $\theta /, / \mathrm{s} / \mathrm{y}$ p, b, v, d, t, c, g, j, l, r, z, s. Los problemas de gramática $(13,5 \%)$ están localizados en la conjugación de verbos y en el uso del género y el número. En cuanto a la ortografía $(3,98 \%)$, cabe destacar los signos de puntuación, la tilde, el orden de los elementos de una oración y la elisión o adición de palabras en el texto.

De igual manera, las notas promedio de los tres años seguidos son menores que la mitad de la nota total (Tabla 10). Se nota que la compresión auditiva es difícil para los estudiantes chinos. Eso se debe a las mismas causas mencionadas en el dictado, como problemas de fonética y problemas de la gramática, las cuales contribuyen a que los estudiantes no puedan obtener informaciones correctas sobre el texto. 
Tabla 11. Notas de la comprensión auditiva de EEE4 de 2009-2011

\begin{tabular}{|l|l|l|}
\hline Año & Nota total & Nota promedio \\
\hline 2009 & 20 & 7,15 \\
\hline 2010 & 20 & 8,32 \\
\hline 2011 & 20 & 6,11 \\
\hline
\end{tabular}

Fuente: Secoeleu (2011) / Elaboración propia

\section{Conclusiones}

Si bien las diferencias entre los dos tipos de exámenes no difieren en su esencia, prevalece en el EEE4 el fuerte apego al método de estudio oficial impartido en los centros de enseñanza pública a través del Español Moderno, frente a la multiplicidad de recursos didácticos que ofrece el DELE. El Español Moderno es el libro de cabecera de los estudiantes y eje central de la carrera. Este método garantiza a los profesores jóvenes, que carecen de la experiencia y la preparación académica requeridas, seguridad para la preparación de sus alumnos para el examen nacional. La proliferación de docentes noveles es el resultado de la gran demanda que ha generado el español en este país asiático.

A pesar de que los responsables de la enseñanza del Español en China no son muy dados a ofrecer datos concluyentes, ya que estos circulan, en muchas ocasiones, a nivel institucional, lo cierto es que el aumento experimentado en el número de alumnos que se presentan al EEE4 en estos últimos años refleja, por un lado, la relevancia que está teniendo el español como LE por los intereses económicos del país (fundamentalmente en Latinoamérica) y, por otro, la proliferación de centros de enseñanza superior que lo incluyen en su oferta académica. Ello no ha supuesto, como podría preverse, una mejora de las estrategias didácticas para su adquisición. Evidencia de esto es el uso del método de enseñanza de Yansheng desde 1999 y la elaboración del EEE4 en función del mismo.

Creemos que el desafío más importante que enfrenta la enseñanza del español en China es la implementación de la investigación en la adquisición de este idioma en las universidades, problema ya planteado por el propio Yansheng, ${ }^{6}$ quien reconoce que son escasas todavía en China las investigaciones en el ámbito de la enseñanza y adquisición del español y se realizan pocos esfuerzos en la formación de los profesores para esta tarea. Ello implica un estancamiento en el acercamiento didáctico y metodológico comparado con otros países.

Si bien hay que reconocer que ha habido aportes importantes en la última década, como la formación de profesores fuera de China en países como España y Cuba; la incorporación de lectores hispanohablantes invitados; las becas para estudiantes destacados, etc., esto no está siendo enfocado para resolver el vacío académico en investigación por la estructura misma de las facultades, que ofrecen pocos incentivos y reconocimiento para participar en conferencias y eventos. No hay que olvidar que el estudio de una lengua extranjera, como desde sus inicios en la República Popular China, es considerado por una gran mayoría como una herramienta práctica y ello ha supuesto que el enfoque de la enseñanza sea asimismo pragmático.

\section{Notas}

1. Colaboradoras: Sofía Wei Xin (魏欣), Cristina Zhu Wen Ying (朱文颖), Cintia Li Hong Xia (李洪霞), Catalina Ma Shi Xu (马世旭), Estela Yu Ling Jiao (于凌蛟), Tonia Tang Na (汤娜). 
2. Entendemos que son alumnos de centros universitarios oficiales, pero no está especificado.

3. Las tablas de este artículo han sido organizadas por los autores.

4. A esta reunión solo asisten docentes chinos.

5. Zhi Chen (2009) aporta este dato y afirma que fueron 1259 alumnos los presentados.

6. Encuentro realizado en La Consejería de Educación de España en China en la ciudad de Pekín sobre "Recursos y materiales en el aula de ele" y organizado por la misma institución los días 2 y 3 de diciembre de 2011. El día 3 contó con la participación de Dong Yan Sheng como ponente principal.

\section{Bibliografía}

Arriaga, N., Blanco, J. M., y Lamarti, R. (2012). La enseñanza del español en China: historia, desarrollo y situación actual (Reseña del libro La enseñanza del español en China: historia, desarrollo y situación actual). SinoELE. http://www.sinoele.org/index.php/ component/content/article?id=156 [Consulta 18 de abril de 2013].

Consejo de Europa (2002). Marco común europeo de referencia para las lenguas: aprendizaje, enseñanza, evaluación. Madrid: Instituto Cervantes.

Chang, F. (2004). ¿Qué estudian los alumnos de español de China? I Encuentro de profesores de español de Asia-Pacífico. [pdf]. http://redined.mecd.gob.es/xmlui/bitstream/ handle/11162/81239/00820113012271.pdf [Consulta 20 de mayo de 2013].

高等学校西班牙语专业四级真题与解析。高等外语专业教学指导委员会西班牙语组编. 2005年5月1日出版。上海外语教育出版社

高等学校西班牙语专业八级考试真题与解析。常福良编。2012年2月1日。上海外语教育出版 社《高等学校西班牙语专业四级考试大纲》, 大纲编写小组组长, 上海外语教育出 版社2011年出版.

Instituto Cervantes. (2007). Manual para los Examinadores. Pekín: Instituto Cervantes.

Ji, F. (2004). Linguistic Engineering in Mao's China: The case of English Language teaching. New Zealand Journal of Asian Studies. 6 (1), 83-99.

Lu, J. (2007). La lengua de Cervantes en la tierra de Confucio. http://congresosdelalengua.es/ cartagena/ponencias/seccion_3/35/jingsheng_lu.htm [Consulta 14 de junio de 2013].

Marco-Martínez, C. y Lee-Marco, J. (2010). La enseñanza del español en China: evolución histórica, situación actual y perspectivas. Cálamo Faspe. 56, 3-14.

Ming, Y. (2004). De la lingüística aplicada a la enseñanza del español. [pdf]. http://www. sinoele.org/images/Revista/2/YangMing.pdf [Consulta 14 de junio de 2013].

《全国高校西班牙语专业教学研究讨会西班牙语教学研讨年会2012》, 编者, 高校外语专 业教学指导委员会西班牙语组.

RedELE. (2012). Entrevista a Lu Jingsheng. Revista. 24. http://www.mecd.gob.es/dctm/ redele/Material-RedEle/Revista/2012/2012_redELE_24_28LuJingsheng. pdf?documentId=0901e72b8144b5eb [Consulta 14 de mayo de 2013].

Sánchez-Griñán, A. (2009). Reconciliación metodológica e intercultural: posibilidades de la enseñanza comunicativa de lenguas en China. marcoELE. revista de didáctica ELE. 8, 1-40. http://dialnet.unirioja.es/servlet/articulo?codigo=3152564 [Consulta 14 de junio de 2013]. 
Sánchez-Griñán, A. (2008). Enseñanza y aprendizaje de español como Lengua Extranjera en China. Retos y posibilidades del enfoque comunicativo. (Tesis doctoral). Universidad de Murcia. http://www.tesisenred.net/handle/10803/10944; jsessionid=B676CFDD61B 96FE711673737CC5F16CF.tdx2 [Consulta 20 de abril de 2013].

Secoeleu《西班牙语专业教学大纲编写组》. 2012, 成都. 全国高校西班牙语教学与专业考试 年会资料汇编。

Secoeleu. (2011) 西安. 全国高校西班牙语教学与专业考试年会资料汇编.

Secoeleu. (2010) 上海. 全国高校西班牙语教学与专业考试年会资料汇编.

Secoeleu. (2009)北京 . 全国高校西班牙语教学与专业考试年会资料汇编.

Secoeleu. (2008) 大连 . 全国高校西班牙语教学与专业考试年会资料汇编.

Secoeleu. (2000). Programa de Enseñanza para Cursos Superiores de las Especialidades de Lengua española en Escuelas Superiores Chinas, 《高校西班牙语专业高年级教学大 纲》. Shanghai: Editorial de Enseñanza de Lenguas Extranjeras de Shanghai.

Secoeleu. (1998). Programa de EnseCñanza para Cursos Básicos de las Especialidades de Lengua Española en Escuelas Superiores Chinas, 《高学西班牙语专业基础阶段教学 大纲》. Shanghai: Editorial de Enseñanza de Lenguas Extranjeras de Shanghai.

Yansheng, D. (2009). Elaboración de material didáctico en China. [pdf]. http://cvc.cervantes. es/ensenanza/biblioteca_ele/publicaciones_centros/pdf/manila_2009/06_plenaria_03. pdf [Consulta 12 de mayo de 2013].

Yansheng, D. y Liu, J. (1999-2008). Español Moderno. (5 vols.). Beijing: Foreign Language Teaching Publications and Research Press.

Zhi, C. (2009). La falta de cohesión y la mentalidad holística de los alumnos chinos. [pdf]. http://cvc.cervantes.es/ensenanza/biblioteca_ele/publicaciones_centros/pdf/ manila_2009/13_investigaciones_06.pdf_[Consulta 14 de junio de 2013]. 
